\title{
A Web Server to Store the Modeled Behavior Data and Zone Information of the Multidisciplinary Product Model in the CAD Systems
}

\author{
Yatish Bathla \\ Doctoral School of Applied Informatics and Applied Mathematics, Óbuda University, Budapest \\ E-mail: yatish.bathla@phd.uni-obuda.hu
}

\author{
Sándor Szénási \\ John von Neumann Faculty of Informatics, Óbuda University, Budapest \\ E-mail: szenasi.sandor@nik.uni-obuda.hu
}

\section{Student paper}

Keywords: web server, multidisciplinary product modeling, rflp structure, cad systems, information retrieval, information storage

Received: January 23, 2019

\begin{abstract}
This work focuses on Human Computer Interaction (HCI) for multidisciplinary product modeling. Requirement Functional Logical Physical (RFLP) structure has emerged as one of the prominent approaches for modeling the multidisciplinary products. To simplify the HCI of an RFLP structured product model, Information Content (IC) provides effective communication and interaction. It controls the RFLP level by the Multilevel Abstraction based Self-Adaptive Definition (MAAD) structure. However, it needs an application to represent the modeled behavior data and zone information of a multidisciplinary product model. Further, the IC application requires an interface to interact with the Computer-Aided Design (CAD) based multidisciplinary product model application and exchange the information between the database of the servers. As per the knowledge of authors, no work has been done yet on the HCI of the IC. Therefore, this paper proposes a Content Web server, which is used to store the modeled behavior data and zone information of the multidisciplinary product model and represented by the IC web application. Then, the Content database is created to store the Layer Info-Chunk (LiC) entities' information of the multidisciplinary product model. Finally, communication between the Content Server and the CAD server is done to represent the IC application interface in the multidisciplinary product application. The Apache Tomcat server, PostgreSQL database, and RESTful web service are used to explain the operations.
\end{abstract}

Povzetek: Pristop HCI temelji na izkoriščanju prednosti človeških možganov in računalniške umetne inteligence. Na ta način so avtorji prispevka izboljšali multidisciplinarno modeliranje oblektov.

\section{Introduction}

A good Human Computer Interaction (HCI) interface in the Computer-Aided Design (CAD) systems deals smartly with the relationship between industrial designers and computer software and hardware, studies the design of manmachine interface model efficiently, the smart design of the virtual interface, multi-user, and multi-sensory interface, and provide a good technical foundation for industrial design [1]. CAD systems simplify the engineering tasks in collecting, using, creating and sharing information, but interface designed without consideration of usability often results in unsatisfied experiences and limited outcomes [2]. Classical Product Models (CPM) in the CAD systems [3] allow product development firms to meet their goals more efficiently. It improves product development time, product quality, productivity and reduces manufacturing as well as product costs. Also, the Requirement Functional Logi- cal Physical (RFLP) structure [4] is applied from the system engineering and offers to handle the multidisciplinary product model as a system. Product assembly is done in the specification tree (white square) of the RFLP structure as shown in Fig. 1. Here, Dassault Systém's CATIA 3DEXPERIENCE [5] is using the RFLP structure for multidisciplinary product modeling. The authors have considered this CAD software for explaining the proposed concepts. There is plenty of research done for improving the HCI of the CAD systems. Some of the appreciated work are as follow:

- A webized interactive CAD review system [12] that uses Super Multi-View (SMV) autostereoscopic displays renders the content through a web browser and handles user interactions via JavaScript. But it is an expensive technology and limited to the CPM.

- A VR (Virtual Reality)-CAD server [13] that embeds 
a commercial CAD engine for loading and modifying native CAD data in a CVE (Collaborative Virtual Environments). It is a distributed architecture that allows collaborative modifications on native CAD data from heterogeneous VR platforms. It is based on the management the $\mathrm{CAD}$ product data and need improvement in terms of visualizing and representation of $3 \mathrm{D}$ product data.

The complexity increases in the case of a multidisciplinary product model as it requires the coordination of huge amounts of model information of the multiple disciplines. Indeed, Information Content (IC) [9] handles the multidisciplinary product model indirectly to record and apply the content of modeled information efficiently [10], which further drives the RFLP level by the Multilevel Abstraction based self Adaptive Definition (MAAD) structure [4]. However, there is a need for Information Content based Web application to represent and store the behavior modeled data [11] from the Process plane [14] and Community Zone information [15] of a multidisciplinary product model.

As a solution, this research work proposes a Content Web Server that consists:

- IC based web application to represent the modeled behavior data and Community zone information of a multidisciplinary product model.

- Content database to store the entities of the modeled behavior data of a multidisciplinary product model.

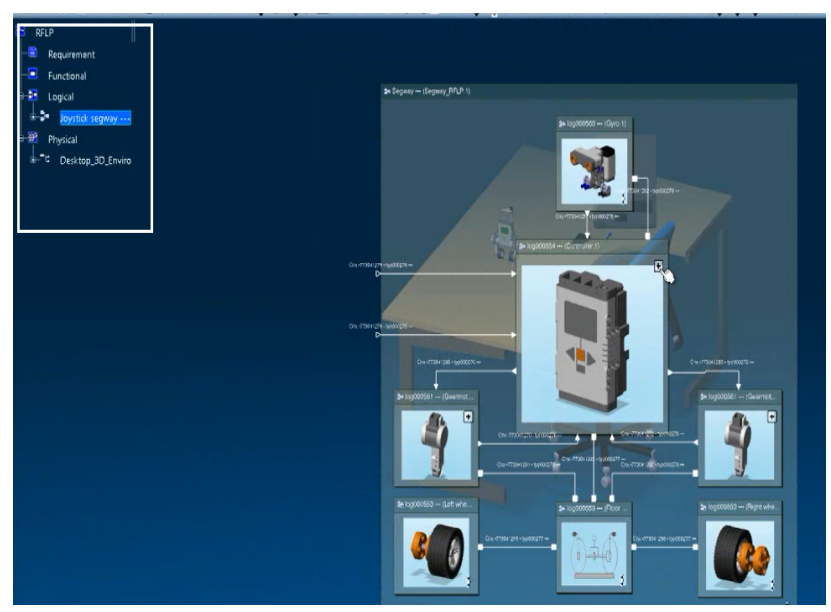

Figure 1: Multidisciplinary Product Model using the RFLP Structure

For the smart interaction of a multidisciplinary product model through the Information Content (IC), an interface is introduced in the multidisciplinary product application through the IC web application. The Apache HTTP Server [16] hosts the IC based web application with the PostgreSQL database [17] to store the multidisciplinary product model data and RESTful web service [18] to exchange the information between the Content and CAD system web server. For the IC web application, the objects collect the Functional and Logical layer information from the InfoChunk [28] entities of the RFLP structure. These objects communicate with the objects of the MAAD structure and collect the modeled behavior data of a multidisciplinary product model. The retrieval of data is according to the process plane of the IC. The objects are based on ObjectOriented Programming (OOP) [8] concepts. The concepts are used frequently in software engineering [29]. The zone and extracted modeled behavior data of a multidisciplinary product are displayed by the IC web application.

This paper starts with the preliminary research where RFLP structured product model, IC, MAAD Structure and InfoChunk entities are discussed. Then human interaction with the IC application and Multidisciplinary application are outlined with the introduction of the Content server. Then, the Content server is explained where the operations and Content database are emphasized. Here, the PostgreSQL database is used for the explanation. Then, Operations of the Content Web server are emphasized. Finally, communication between the Content and CAD system web server is elaborated. Here, the RESTful API web service is used for the explanation.

\section{Background}

The product modeling is the prominent field. There are plenty of companies like Dassault Systémes [19], Autodesk [20], Robert McNeel [21], Pixologic [22] investing a lot of money in this market. The feature driven CPM (Classical Product Model) [7] is most commonly used for discipline specific product modeling. CPM is limited to the physical level. Handling a complex product model is a challenging task due to the involvement of a large number of engineering objects and their relationship. But, product modeling is not limited to the physical layer. The separated or only slightly integrated mechanical engineering modeling increasingly demanded multidisciplinary integration [30]. Modeling of a multidisciplinary product must have a means for the integration of discipline specific models into a model with a unified structure. Higher abstraction is realized by using of RFLP structure based product model [4]. It is commonly used for multidisciplinary modeling as it models the product as a system. It is compliant with the IEEE 1220 standard. This structure has four layers i.e. Requirement layer for the requirements against the product, Function layer for the functions to fulfill requirements, Logical layer for the product wide logical connections, and Physical layer for the representations of physically existing objects. It accommodates product behavior definitions on its Functional and Logical levels. In the RFLP structure of the Dassault Systém's CATIA 3DEXPERIENCE software, Dymola [6] is used to analyze the dynamic logical behavior of a product and Modelica [7] is used for logical and physical modeling of the technical system. Modelica is a 
multi-domain modeling language for component-oriented modeling of complex systems and based on the OOP concepts. [30].

Information Content (IC) [9] assists effective communication in the multidisciplinary product modeling. It drives the RFLP level by the MAAD structure. The MAAD modeling [31] methods and model structures are introduced as generalized means for the support of higher level abstraction based generation of RFLP elements. The MAAD modeling was based on the knowledge representation, contextual change propagation, and extended feature definition capabilities for advanced modeling systems. In the IC, the intent is defined by the human to control the definition of engineering objects of a product model[32]. In the Engineering objectives layer, the Process plane [14] is used to store the processes performed on a multidisciplinary product model. Also, Community zones [15] are used by the IC to organize the complex product model entities and their relationship as shown in Fig. 2. Here, product model space is divided into community zones based on the discipline, specification or configuration. In this figure, the multidisciplinary product model is divided into community Internal or External based on configuration. The information of the process plane and community zones are shown on the representation plane of the IC.

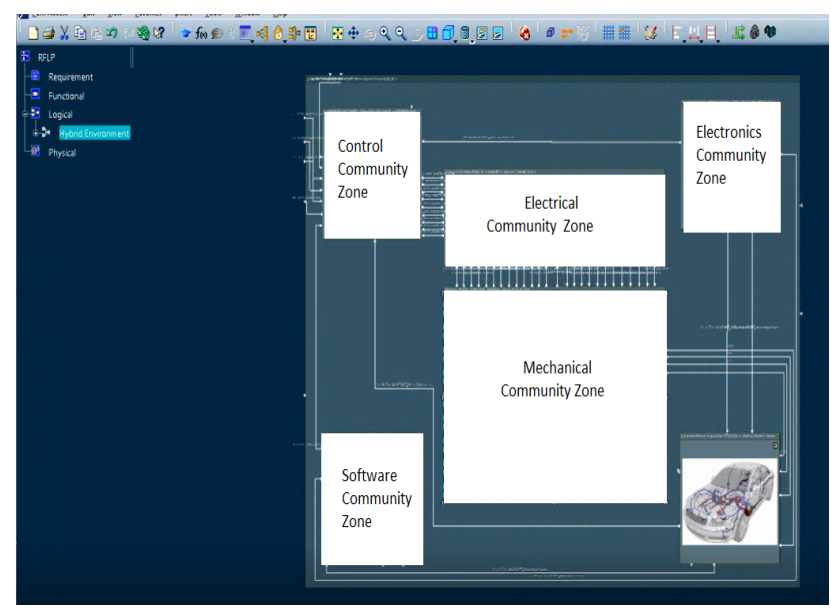

Figure 2: Community Zones in the RFLP Structure

Layer Info-Chunk (LiC) [28][38] entities were introduced in the Functional and Logical layer of the RFLP structure for effective communication with the IC. It controls the behavior data activities of the RFLP structure. The Logical layer Info-Chunk (LiCL) entity stores the information of the Logical layer and the Component Info-Chunk (CiC) entity stores the information of the Logical component. Further, the Functional layer Info-Chunk (LiCF) entity stores the main function information of the Functional layer and the Sub-Function Info-Chunk (SFiC) entity stores the subfunction information of a function. Considering the above mentioned concepts as a base, the authors propose the effective Human Computer Interaction (HCI) for the multi- disciplinary product modeling by using the IC application.

\section{User interaction and multidisciplinary product application}

In this research work, the Multidisciplinary product model is handled and controlled through the IC application. IC application is a web based application in the JSP (JavaServer Pages) format [23]. It resides on a web server called Content Server. It is explained in the next section. A multidisciplinary product model application using the RFLP structure is an application in the 3DXML format [24]. The 3DEXPERIENCE [25] CAD software requires ENOVIA [26] Product Lifecycle Management (PLM) system in the backend that allows the data to be stored in one central location, therefore, access from anywhere. ENOVIA V6 uses Microsoft SQL Server 2008 R2 Enterprise platform for database management [39]. Therefore, in this research work, CAD Product server refers to the Microsoft SQL Server [27]. The IC, MAAD Structure and LiC entities in the RFLP structure communicate through the Info-Chunk objects, which is based on the Object-Oriented Programming Principles (OOP) [8]. The advantage of the IC application is a simpler user interface and efficient organization of objects retrieved from the product model. There are two scenarios to be considered:

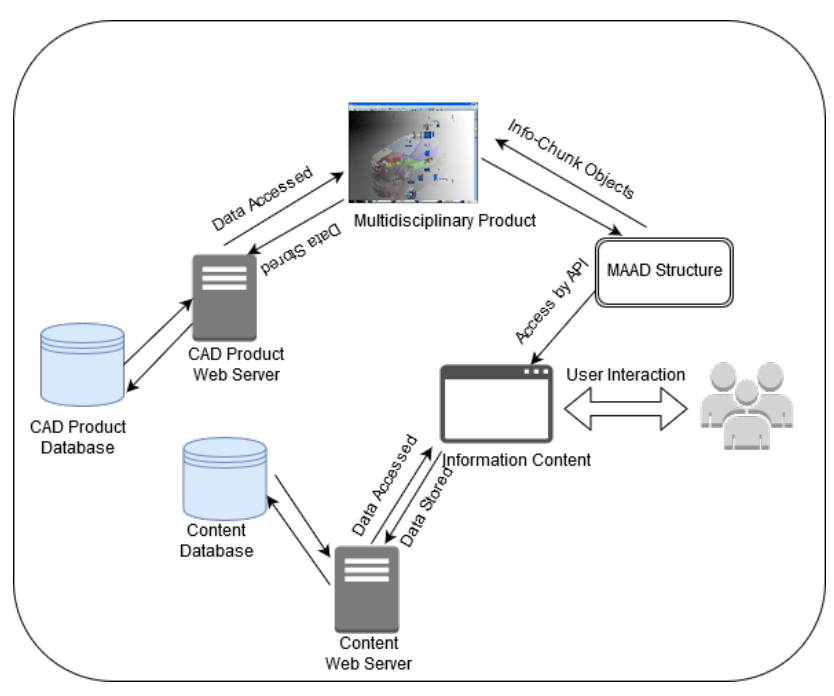

Figure 3: User Interaction from the Information Content application

- The user interacts with the IC application to access the Multidisciplinary product application as shown in Fig. 3. IC drives the RFLP structure through the MAAD structure. Every application has its own web server for the resource management and database to locate the information. The database of the CAD 
product server is isolated from the database of the content server.

- The user interacts with the Multidisciplinary product application to access the IC application through a separate plane as shown in Fig. 4. There is an interface between the two applications. The database of the CAD product server retrieves the process and zone partition information using the web services from the database of the content server.

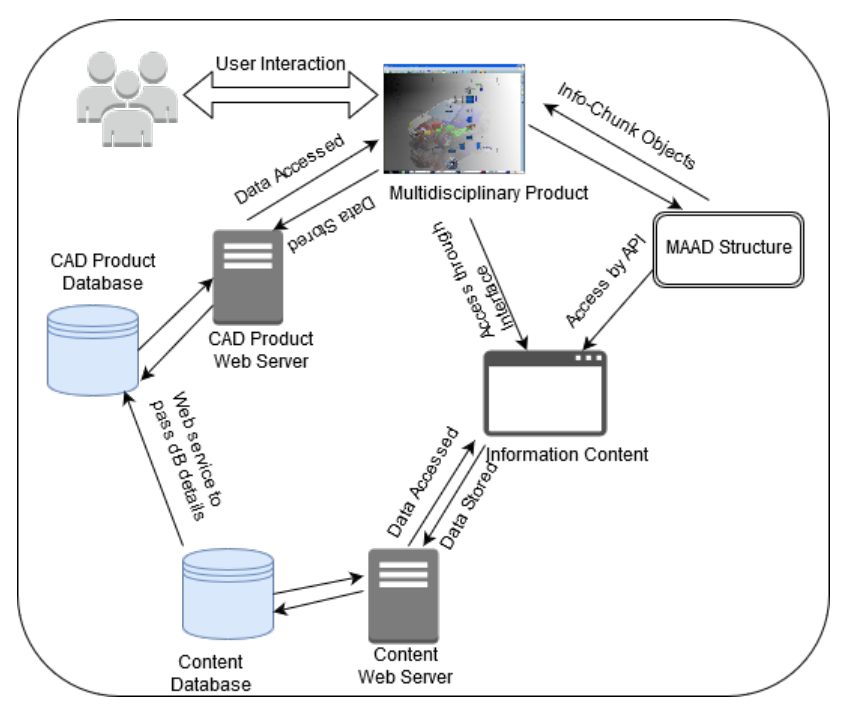

Figure 4: User Interaction from the Multidisciplinary product application

\section{Content web server}

Content Web server is the Apache Http Server [33] that used to store and display the data of the Information Content (IC) as shown in Fig. 5. The Tomcat Servlet [34] is used for the Information Content web application. Enterprise Management Agent (EMA) is the integral software component responsible for managing and maintaining the IC based Web application. It also allows monitoring the CAD Product database, through management plug-ins and connectors. The Process partition consists of the outcome of the process plane of the IC, the product model after a certain set of the process applied and the files that explain the location of outcomes of the process plane and product model. Similarly, the Zone partition consists of the outcome of the community zone of the IC, the product model after divided into the zones and the files that explain the location of outcomes of the community zone and product model. The outcomes are the graphs obtained from the Process plane and Community zones. The authors store the graphs in the PNG format [35], product model application in the Dassault Systém's 3DEXPERIENCE file format (3DXML) [36], XML file format [37] for the data inter- change and SCN file format for the 3D product model (Assembly model or part model) management.

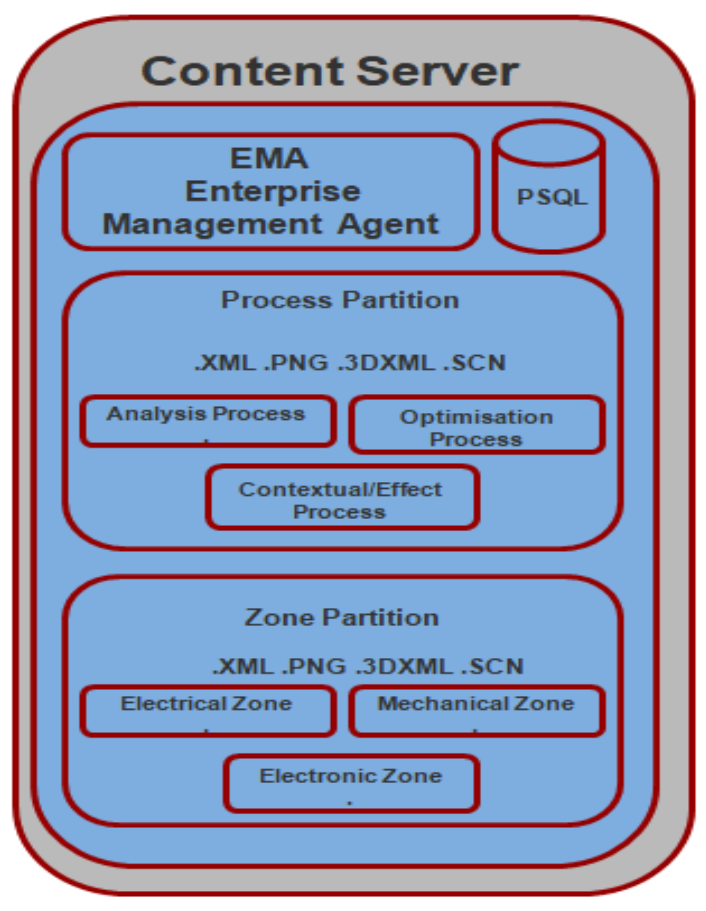

Figure 5: Content Server

The Content database is created by using the PostgreSQL. It stores the data of the Information Content application while handling the behavior modeled data and zone information of the multidisciplinary product application. Entity Relationship (ER) [40] diagram is used for the physical data modeling as shown in Fig. 6. It is required for the schema level for creating a database. There are nine tables created based on the concept of LiC entities of the RFLP structure. During the product modeling using the RFLP structure, there is a set of information transferred from the Requirement layer to the Physical layer. Behaviors of a product model are represented in the Functional and Logical layer of the RFLP structure. LiCF table is used to store the attributes of the Functional layer and the LiCL table is used to store the attributes of the Logical layer of the $\mathrm{LiC}$ (Layer Info-Chunk) entity of the RFLP structure. In these tables, some of the data types are built-in while others are user-defined. In the case of the LiCL table,

- LiCLConnector is the Enumerated data type that stores the inner and connector values of the LiCL entity.

- LiCF, CiC, and LiCLDataModel are the composite data type, whose attributes and data types are specified in the Content ER diagram.

- In the CiC table, CiCConnector is the Enumerated data type that stores the inner and stream values of the CiC entity 


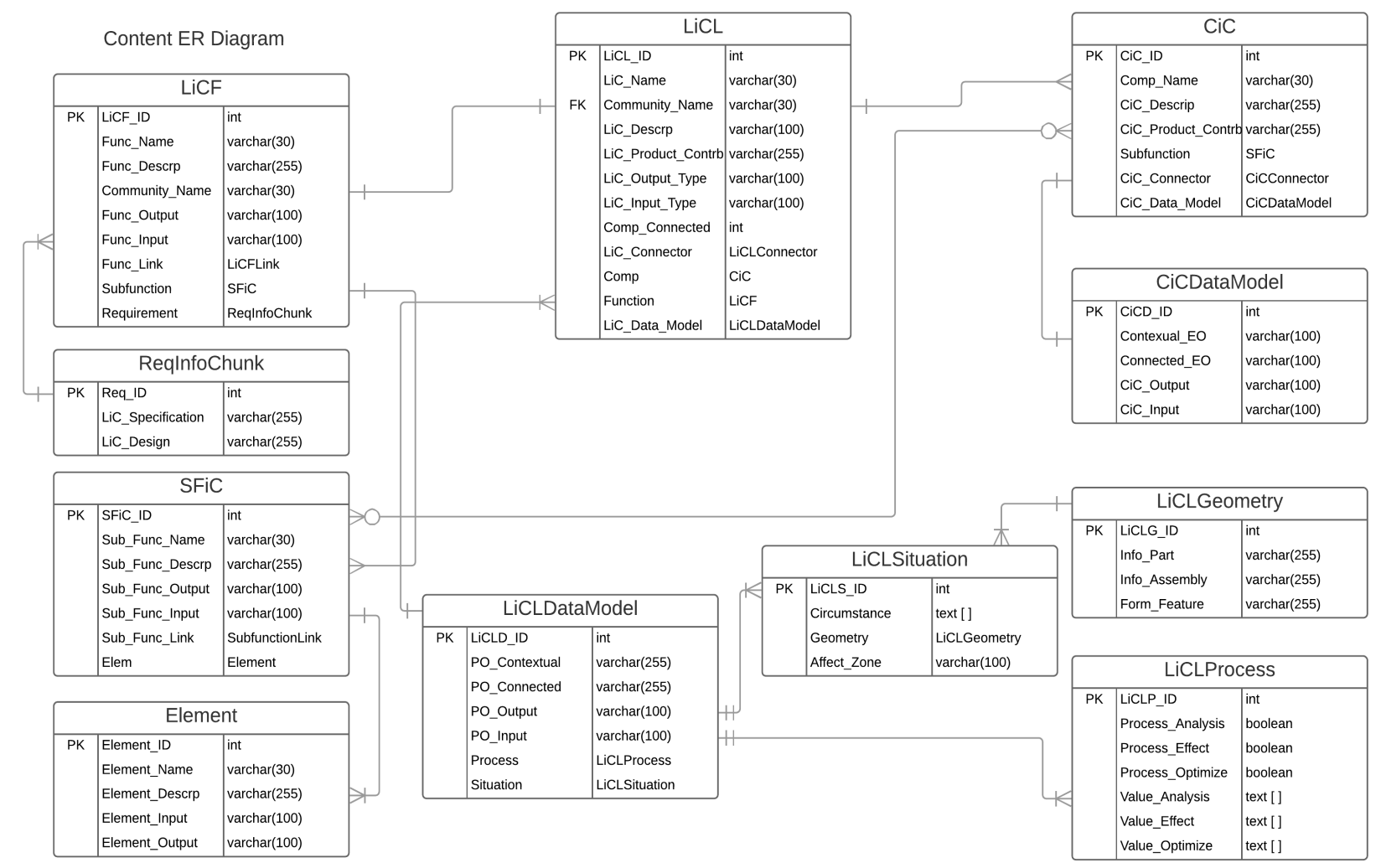

Figure 6: Content Entity Relationship Diagram.

- In the CiC table, SFiC and CiCDataModel is the composite data type, whose attributes and data types are specified in the Content ER diagram.

- In the LiCLDataModel table, LiCLSituation and LiCLProcess are the user-defined composite data types.

The CiC table is used to store the attributes of the $\mathrm{CiC}$ (Component Info-Chunk) entity present in a LiCL entity. LiCLDataModel table is used to store the attributes of the detailed description of the Physical layer of the RFLP structure. LiCLProcess table is used to store the attributes of the Process plane of the IC. LiCLSituation table is used to store the attributes of a situation in the logical layer of the RFLP structure. Here, LiCLGeometry composite data type is used to store the information of a part model or assembly model in a situation. In the case of the LiCF table,

- LiCFLink is the Enumerated data type that stores the inner and connector values of the LiCF entity.

- SFiC and ReqInfoChunk are the composite data types, whose attributes and data types are specified in the Content ER diagram.

- In the SFiC table, SubFunctionLink is the Enumerated data type that stores the inner and stream values of the SFiC entity
- In the SFiC table, Element is the composite data type, whose attributes and data types are specified in the Content ER diagram.

- In the ReqInfochunk table, attributes and data types are specified in the Content ER diagram

For reference, LiCLConnector, LiCLDataModel, and LiCLProcess commands are demonstrated using the SQL statements of PostgreSQL as shown below. Here, new tables and data type is created using the CREATE statement.

CREATE TYPE LiCLConnector AS ENUM (' inner', 'Stream');

CREATE TYPE LiCLDataModel AS ( LiCLD_ID INT,

PO_Contextual VARCHAR(255), PO_Connected VARCHAR(25 5$)$,

PO_Output VARCHAR(100),

PO_Input VARCHAR( 100$)$,

Process LiCLProcess,

Situation LiCLSituation);

CREATE TYPE LiCLProcess AS (

LiCLP_ID INT,

Process_Analysis BOOLEAN, 


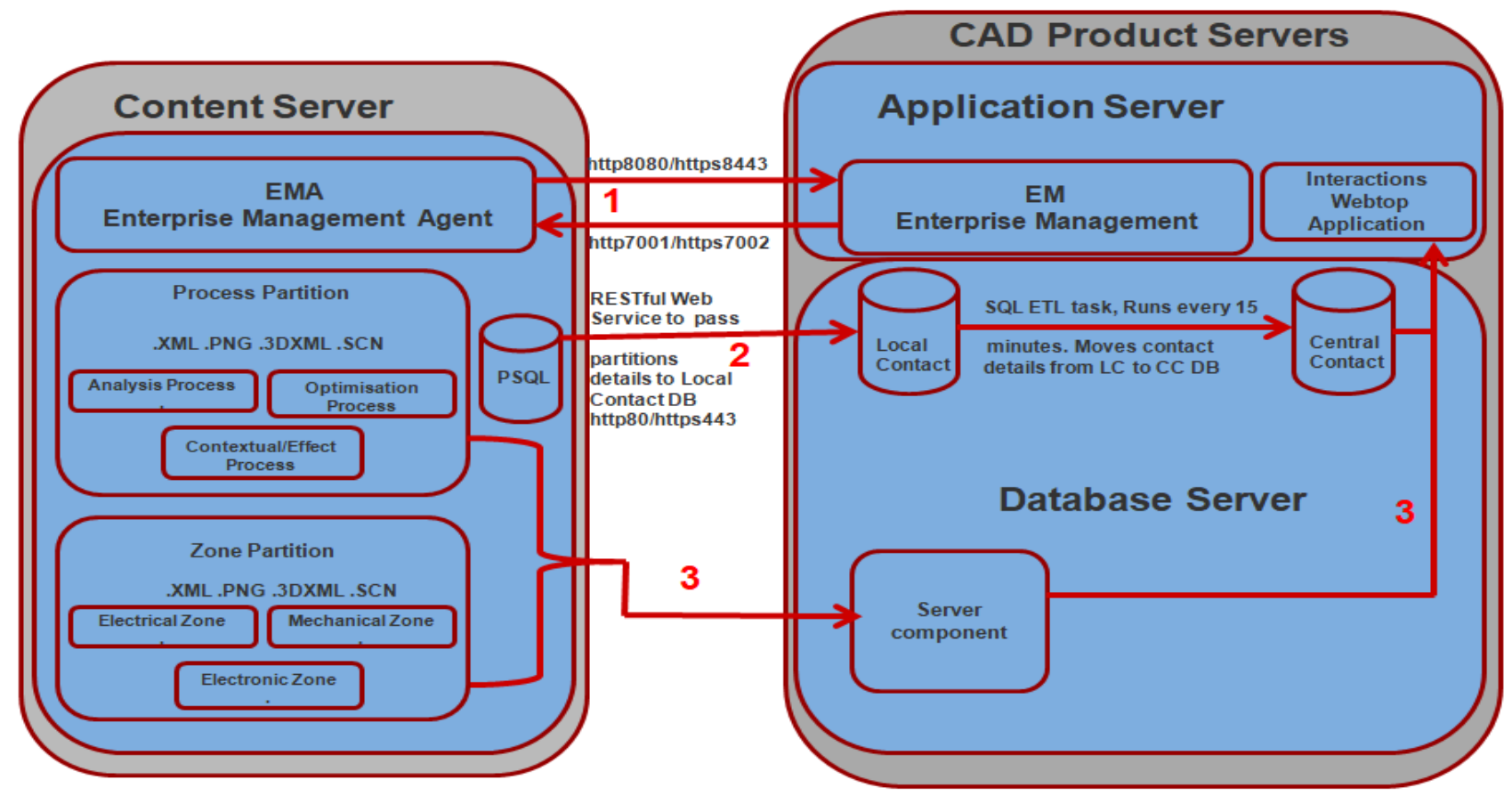

Figure 7: Communication between Content server and CAD server.

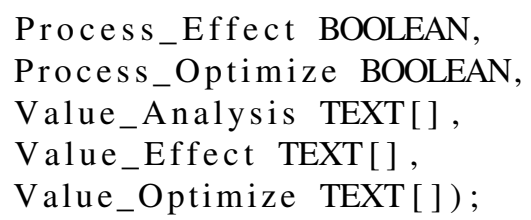

The modeled behavior data of a multidisciplinary product is stored in the entities based on the entities' relationship. The entities are populated by the IC application.

- In the context of the Functional layer, One LiCF entity may have many SFiC entities and one or many LiCF entities may have one ReqInfoChunk entity. Further, one SFiC entity may have one or many Element entities.

- In the context of the Logical layer, One LiCL entity may have one $\mathrm{LiCF}$ entity and many $\mathrm{CiC}$ entities. Also, one or many LiCL entities may have one LiCLDataModel entity. Further, one and only one LiCLDataModel entity may have one or many LiCLProcess and LiCLSituation entities. Here, one or many LiCLSituation entities may have one LiCLGeometry entity. Also, One CiC entity may have one CiCDataModel.

\subsection{Operations}

A human expert handles the multidisciplinary application through the IC application. To model the behavior data, the process plane from the Engineering objectives layer of the IC interacts with the Info-Chunk objects of the Product Behaviors level of the MAAD structure, which further, drives the Info-Chunk objects of the Functional and Logical layer of the RFLP structure. Here, the Process plane of the IC communicates with LiC entities of the RFLP structure using the Info-Chunk objects to retrieve the modeled behavior data of a multidisciplinary product plane. The data is stored in the Process partition. Also, the Product model is divided into community zone based on the discipline. The outcome is stored in the Zone partition. Then, a human interacts with the results stored in the partition through the representation plane of the Interactive IC application. The outcome could be static or dynamic and represented as graphs, images or animation.

\subsection{Communication between content server and CAD product server}

The CAD Server pulls process partition and zone partition from the Content server when replaying through the IC interface in the Multidisciplinary web application as shown in Fig. 7. Content server partitions information is saved in CAD server cache and auto deleted almost immediately after a replay. EM-EMA Link handles publishing of configuration between framework and Content server. RESTful Web API Link handles passing of modeled behavior data and zone partition details from Postgres job queue to Local Contact DB which then gets moved on to Central Contact DB by ETL SQL process of the CAD server. The advantage of this API there is no need to install additional software or libraries and provide a great deal of flexibility. Content Server handles retrieval of .XML, .PNG, .3DXML and .SCN content from the Content server to IC Webtop 
application [41] interface of the Multidisciplinary application for replay. The process and zone partition details are taken from Central Contact DB and converted to .3DXML format for the multidisciplinary application and then it is deleted.

\section{Conclusion}

This research work proposes the Content server to store zone and modeled behavior information of a multidisciplinary product model. This work starts with the Human Computer Interaction (HCI) of a multidisciplinary product model where the model is handled directly by the Information Content (IC) web application or through an interface in the multidisciplinary product application. The operation and process of IC web application are stored in the Content server. Then, the server is explained in brief, where data is stored in the Zone partition and Process partition based on the communication between the IC and RFLP structure. It is done by the Info-Chunk objects and stored in the Content database. Finally, communication between the Content Server and CAD product server is explained where information of zone partition and process partition pushed temporarily to the CAD product server so that IC webtop application in the main application could handle the multidisciplinary product model. As Modelica and Info-Chunk objects are based on the OOPS concepts, the RFLP structure and IC could be compatible with each other and exchange information easily. This research work is an effort to provide efficient user interaction of a multidisciplinary product model through the Information Content.

\section{Acknowledgement}

This study is sponsored by the Doctoral School of Applied Informatics and Applied Mathematics, Óbuda University, Budapest, Hungary and Tempus foundation. The authors gratefully acknowledge to his supervisor Dr. Horváth László for the guidance to write this paper.

\section{References}

[1] Z. Liang, Z. Jian, Z. Li-Nan and L. Nan. The Application of Human-Computer Interaction Idea in Computer Aided Industrial Design. International Conference on Computer Network, Electronic and Automation (ICCNEA), 160-164, 2017.https://doi.org/10.1109/iccnea.2017.71

[2] Li, Yujiang, Mikael Hedlind, and Torsten Kjellberg. Usability evaluation of CAD CAM: State of the art. Procedia CIRP, 36, 205-210, 2015. https://doi.org/10.1016/j.procir.2015.01.053

[3] Bloom, R. Getting started with CAD/CAM. Materials \& Design, 17(4): 223-224,
1996.https://doi.org/10.1016/S0261-3069(97)889303.

[4] L. Horváth and I. J. Rudas. Systems engineering methods for multidisciplinary product definition. IEEE 12th International Symposium on Intelligent Systems and Informatics (SISY), 293-298, 2014. https://doi.org/10.1109/sisy.2014.6923604

[5] Barfield, W. The law of virtual reality and increasingly smart virtual avatars. Research Handbook on the Law of Virtual and Augmented Reality, 2-4, 2018. https://doi.org/10.4337/9781786438591.00008

[6] Dempsey, M. Dymola for Multi-Engineering Modelling and Simulation. IEEE Vehicle Power and Propulsion Conference, 1-6, 2006. https://doi.org/10.1109/vppc.2006.364294

[7] Peter Fritzson. Principles of Object-Oriented Modeling and Simulation with Modelica 3.3: A Cyber-Physical Approach, WileyIEEE Press, John Wiley \& Sons Inc, 2015. https://doi.org/10.1002/9781118989166

[8] Baesens, B., Backiel, A., \& Broucke, S.vanden (Eds.). Beginning Java Programming, Wrox, 2012. https://doi.org/10.1002/9781119209416

[9] L. Horváth and I. J. Rudas. Towards the Information Content-driven Product Model. Proceedings of the IEEE International Conference on System of Systems Engineering, 1-6, 2008. https://doi.org/10.1109/sysose.2008.4724183

[10] Laszlo Horvath, Imre J. Rudas. Bringing up product model to thinking of engineer. Proceedings of the IEEE International Conference on Systems, Man and Cybernetics, 1355 - 1360, 2008. https://doi.org/10.1109/icsmc.2008.4811474

[11] L. Horváth and I. J. Rudas. Elevated level design intent and behavior driven feature definition for product modeling. 39th Annual Conference of the IEEE Industrial Electronics Society, 1(2), 4374-4379, 2013. https://doi.org/10.1109/iecon.2013.6699839

[12] Seo, Daeil, Yongjae Lee and Byounghyun Yoo. Webizing Interactive CAD Review System Using Super Multiview Autostereoscopic Displays. HCII Posters, Part II, CCIS 714, 62-67, 2017. https://doi.org/10.1007/978-3-319-58753-0-10

[13] Okuya, Yujiro, Nicolas Ladeveze, Olivier Gladin, Cédric Fleury and Patrick Bourdot. Distributed Architecture for Remote Collaborative Modification of Parametric CAD Data. IEEE Fourth VR International Workshop on Collaborative Virtual Environments (3DCVE), 1-4, 2018. https://doi.org/10.1109/3dcve.2018.8637112 
[14] Yatish Bathla. Different types of process involved in the information content product model. Proceedings of the IEEE 14th International Symposium on Intelligent Systems and Informatics (SISY), 99-104, 2016. https://doi.org/10.1109/sisy.2016.7601478

[15] Yatish Bathla. Structured organization of Engineering Objects in the information content of PLM system. Proceedings of the IEEE 11th International Symposium on Applied Computational Intelligence and Informatics (SACI), 473 - 478, 2016. https://doi.org/10.1109/saci.2016.7507424

[16] Fielding, R.T., Kaiser, G. The Apache HTTP Server Project, IEEE Internet Computing, 1(4): 88 - 90, 1997. https://doi.org/10.1109/4236.612229.

[17] Neil Matthew, Richard Stones. Introduction to PostgreSQL. Apress, 2005, https://doi.org/10.1007/9781-4302-0018-5.

[18] Li Li and Wu Chou. Design Patterns for RESTful Communication Web Services. IEEE International Conference on Web Services, 2010. https://doi.org/10.1109/icws.2010.101

[19] Smolek, P., Heinzl, B., Ecker, H., \& Breitenecker, F.. Exploring the Possibilities of CoSimulation with CATIA V6 Dynamic Behavior Modeling, SNE Simulation Notes Europe, 23(3-4), 2013. https://doi.org/10.11128/sne.23.sn.10205

[20] Sha Liu. Sustainable Building Design Optimization Using Building Information Modeling. ICCREM, 2015. https://doi.org/10.1061/9780784479377.038

[21] Chen, H., Lowe, A.A., de Almeida, F.R., Wong, M., Fleetham, J.A., Wang, B.. Threedimensional computer-assisted study model analysis of long-term oral-appliance wear. Part 1: Methodology. American Journal of Orthodontists \& Dentofac. Orthop, 134(3): 393-407, 2008. https://doi.org/10.1016/j.ajodo.2006.10.030

[22] Tim Vernon. Zbrush. Journal of Visual Communication in Medicine, 34(1): 31-35, 2011. https://doi.org/10.3109/17453054.2011.548735

[23] Lennart Jörelid. J2EE FrontEnd Technologies: A Programmer's Guide to Servlets, JavaServer Pages, and Enterprise JavaBeans. Springer, 2002. https://doi.org/10.1007/978-1-4302-1148-8

[24] Jing Chen, Jiawei Li, Mo Li. Progressive Visualization of Complex 3D Models Over the Internet. Transactions in GIS, 20(6): 887-902, 2016. https://doi.org/10.1111/tgis.12185

[25] Adam Suydam, Jason Pyles. Lockheed Martin Conceptual Design Modeling in the Dassault Systemes 3DEXPERIENCE Platform, AIAA Scitech Forum, 2020. https://doi.org/10.2514/6.2020-1391
[26] Ling-Long Lin, Yun-Tao Song, Yu-Xiang Tang, Qing-Qing Du, Yi-Peng Gong. Implementation and application study on 3D collaborative design for CFETR based on ENOVIA VPM. Fusion Engineering and Design, 100, 198-203, 2015. https://doi.org/10.1016/j.fusengdes.2015.05.072

[27] Buffington, J.. Microsoft SQL Server. Data Protection for Virtual Data Centers. 2011, https://doi.org/10.1002/9781118255766.ch8

[28] Yatish Bathla. Conceptual Models of Information Content for Product Modeling. Acta Polytechnica Hungarica, XV (2): 169-188, 2018. https://doi.org/10.12700/aph.15.1.2018.2.9

[29] H. F. Krikorian. Introduction to objectoriented systems engineering.1. Journal of IT Professional. V(2), 38-42, 2003. https://doi.org/10.1109/MITP.2003.1191791

[30] L. Horváth. New method for enhanced driving of entity generation in RFLP structured product model. 12th IEEE Conference on Industrial Electronics and Applications (ICIEA), 541-546, 2017. https://doi.org/10.1109/iciea.2017.8282903

[31] L. Horváth and I. J. Rudas. Multilevel Abstraction Based Self Control Method for Industrial PLM Model. IEEE International Conference on Industrial Technology, 695-700, 2014. https://doi.org/10.1109/icit.2014.6894915

[32] Laszlo Horvath. New methods on the way to intelligent modeling in computer integrated engineering. Proceedings of the 36th Annual Conference on IEEE Industrial Electronics Society(IECON), 1359-1364, 2010. https://doi.org/10.1109/iecon.2010.5675486

[33] Fielding, R.T., Kaiser, G.. The Apache HTTP Server Project. IEEE Internet Computing, 1(4): 88-90, 1997. https://doi.org/10.1109/4236.612229.

[34] Aleksa Vukotic, James Goodwill. Apache Tomcat 7, Springer Nature Switzerland, Apress 2011. https://doi.org/10.1007/978-1-4302-3724-2

[35] Randers-Pehrson, G., Boutell PNG (Portable Network Graphics) Specification, Version 1.0. PNG Dev. Gr., 1999. https://doi.org/10.17487/rfc2083

[36] Roberto Riascos, Laurent Levy, Josip Stjepandić, Arnulf Fröhlich. Digital Mock-up. Concurrent Engineering in the 21st Century Foundations, Developments and Challenges, 355-388, 2015. https://doi.org/10.1007/978-3-319-13776-6_13

[37] Clarke, K.S.. Extensible markup language (XML), in: Understanding Information Retrieval Systems. Management, Types, and Standards, 2011. https://doi.org/10.1201/b11499-52 
[38] Yatish Bathla. Info-Chunk driven RFLP Structure based Product Model for Multidisciplinary Cyber Physical Systems. IEEE 16th International Symposium on Intelligent Systems and Informatics (SISY), pp. 000327-000332, 2018. https://doi.org/10.1109/sisy.2018.8524653

[39] He Youquan, Zhang Wei, Xie Jianfang, Wang Jian, Qiu Hanxing. Integrated Application of PLM Based ENOVIA Platform in Domestic Manufacturing Industry. International Conference on Information Management, Innovation Management and Industrial Engineering, nov, 2011. https://doi.org/10.1109/iciii.2011.202

[40] Richard Earp, Sikha Bagui. Database Design Using Entity-Relationship Diagrams. Foundations of Database Design, june, 2003. https://doi.org/10.1201/9780203486054

[41] Heller, D., Krenzelok, L., Orr, J. Webtop: Realities in designing a web-application platform. IEEE Potentials, Proceedings of the 2003 Conference on Designing for User Experiences. DUX '03, 2003, https://doi.org/10.1145/997078.997115 
